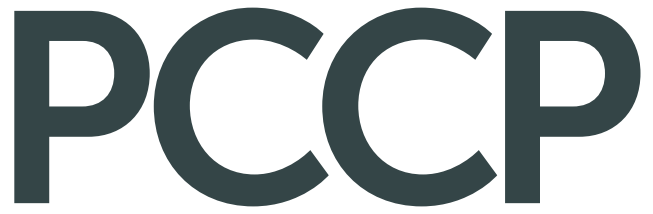

Physical Chemistry Chemical Physics

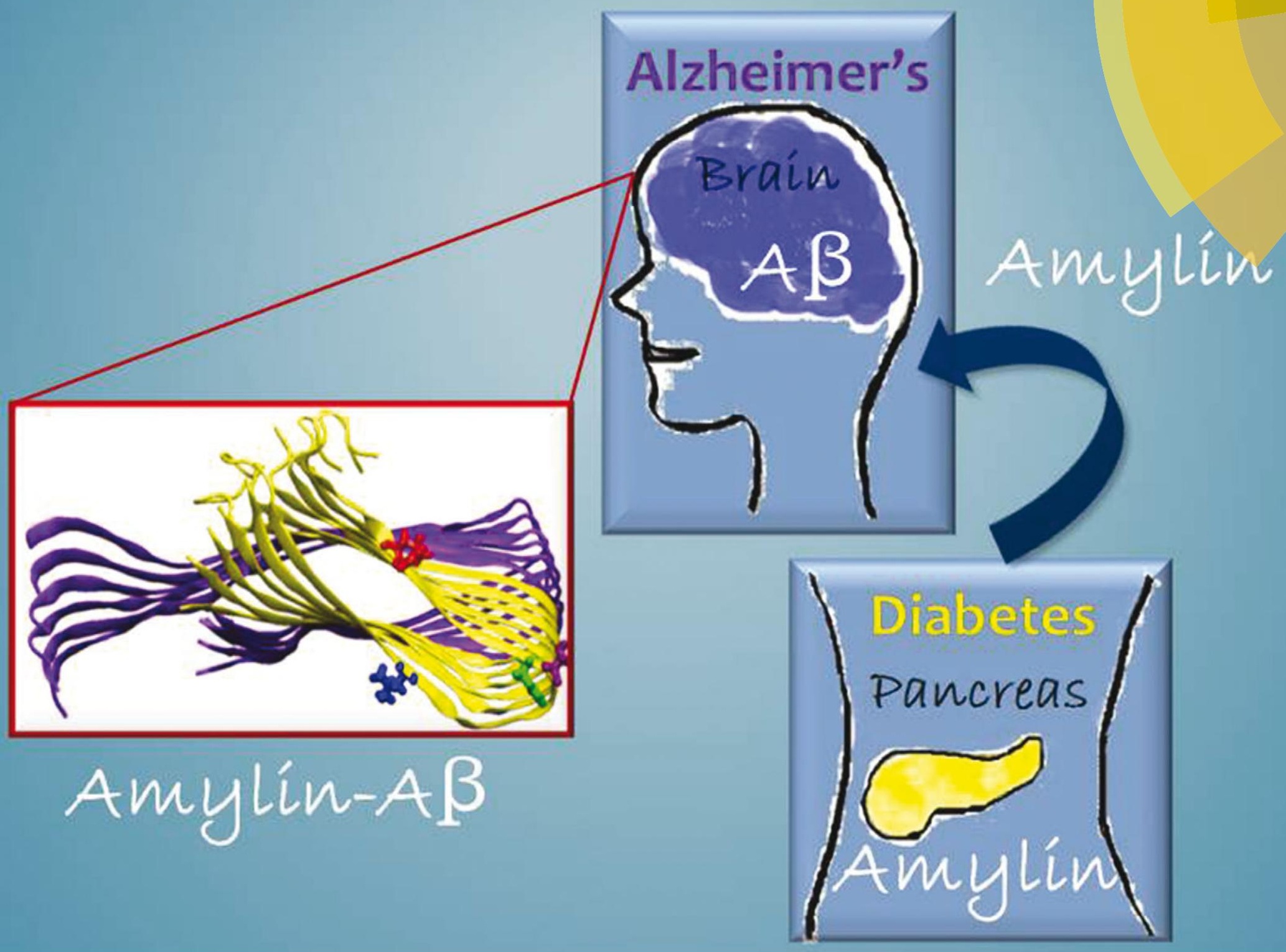

ISSN 1463-9076

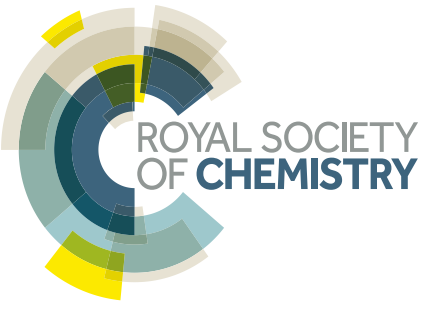

\section{PAPER}

Ruth Nussinov, Yifat Miller et al.

Amylin-A $\beta$ oligomers at atomic resolution using molecular dynamics simulations: a link between Type 2 diabetes and Alzheimer's disease

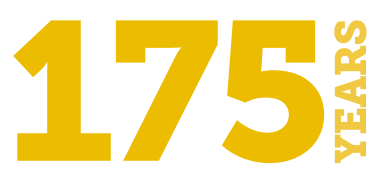




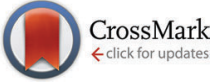

Cite this: Phys. Chem. Chem. Phys., 2016, 18, 2330

Received 9th June 2015 Accepted 24th August 2015

DOI: $10.1039 / c 5 c p 03338 a$

www.rsc.org/pccp

\title{
Amylin-A $\beta$ oligomers at atomic resolution using molecular dynamics simulations: a link between Type 2 diabetes and Alzheimer's disease $\dagger$
}

\author{
Michal Baram, ${ }^{a b}$ Yoav Atsmon-Raz, ${ }^{a b}$ Buyong Ma, ${ }^{c}$ Ruth Nussinov*cd and \\ Yifat Miller*ab
}

\begin{abstract}
Clinical studies have identified Type 2 diabetes (T2D) as a risk factor of Alzheimer's disease (AD). One of the potential mechanisms that link T2D and AD is the loss of cells associated with degenerative changes. Amylin $_{1-37}$ aggregates (the pathological species in T2D) were found to be co-localized with those of $A \beta_{1-42}$ (the pathological species in $A D$ ) to form the Amylin ${ }_{1-37}-A \beta_{1-42}$ plaques, promoting aggregation and thus contributing to the etiology of $A D$. However, the mechanisms by which Amylin ${ }_{1-37} \mathrm{CO}_{\text {-aggregates }}$ with $A \beta_{1-42}$ are still elusive. This work presents the interactions between Amylin ${ }_{1-37}$ oligomers and $A \beta_{1-42}$ oligomers at atomic resolution applying extensive molecular dynamics simulations for relatively large ensemble of cross-seeding Amylin ${ }_{1-37}-A \beta_{1-42}$ oligomers. The main conclusions of this study are first, $A \beta_{1-42}$ oligomers prefer to interact with $A m y l i n_{1-37}$ oligomers to form single layer conformations (in-register interactions) rather than double layer conformations; and second, in some double layer conformations of the cross-seeding Amylin ${ }_{1-37}-A \beta_{1-42}$ oligomers, the Amylin ${ }_{1-37}$ oligomers destabilize the $A \beta_{1-42}$ oligomers and thus inhibit $A \beta_{1-42}$ aggregation, while in other double layer conformations, the Amylin $1-37$ oligomers stabilize $A \beta_{1-42}$ oligomers and thus promote $A \beta_{1-42}$ aggregation.
\end{abstract}

\section{Introduction}

Type 2 diabetes (T2D) is one of the most common metabolic disorders and its prevalence increases with age. Clinical and epidemiological studies identified T2D as a risk factor of Alzheimer's disease (AD). ${ }^{1-3}$ Several studies have shown that there are many similarities between $\mathrm{T} 2 \mathrm{D}$ and $\mathrm{AD}$, and that both conditions underlie common physiological processes. ${ }^{3} \mathrm{AD}$ is characterized by intracellular neurofibrillary tangles (NFTs), containing an abnormally hyperphosphorylated form of tau protein, and extracellular senile plaques, mainly composed of Amyloid $\beta(A \beta)$ aggregates. Both Tau and $A \beta$ aggregates which are the pathological hallmarks of $\mathrm{AD}$ are found in $\mathrm{T} 2 \mathrm{D} .^{4,5}$

\footnotetext{
${ }^{a}$ Department of Chemistry, Ben-Gurion University of the Negev, Beér-Sheva 84105, Israel

${ }^{b}$ Ilse Katz Institute for Nanoscale Science and Technology, Ben-Gurion University of the Negev, Beér-Sheva 84105, Israel. E-mail: ymiller@bgu.ac.il; Tel: +972-86428705

${ }^{c}$ Basic Science Program, Leidos Biomedical Research, Inc., Cancer and Inflammation Program, National Cancer Institute, Frederick, MD 21702, USA. E-mail: NussinoR@helix.nih.gov; Tel: +1 3018465579

${ }^{d}$ Sackler Inst. of Molecular Medicine, Department of Human Genetics and Molecular Medicine, Sackler School of Medicine, Tel Aviv University, Tel Aviv 69978, Israel

$\dagger$ Electronic supplementary information (ESI) available. See DOI: 10.1039/c5cp03338a
}

One of the potential mechanisms that link T2D and AD is the loss of cells associated with degenerative changes. ${ }^{1,2,6} \mathrm{AD}$ is a neurodegenerative disease with extensive neuronal loss resulting from Tau and $A \beta$ aggregation. T2D is also a degenerative disease that results from selective destruction of pancreatic $\beta$-cells and associated neuropathies, ${ }^{7-9}$ which are caused by aggregation of the neuroendocrine hormone named "Amylin".

Recently, Jackson et al. ${ }^{10}$ identified Amylin deposits in the temporal lobe gray matter - a major component of the central nervous system - of diabetes patients. In addition to the Amylin deposition in the human brain, Amylin aggregates are co-localized with $\mathrm{A} \beta$ aggregates to form the Amylin- $\mathrm{A} \beta$ plaques, promoting aggregation and thus contributing to the etiology of AD. Recent in vivo studies investigated the cross-seeding between $A \beta$ and Amylin aggregates. ${ }^{11-13}$ Yet, the mechanisms by which Amylin co-aggregates with $A \beta$ are still elusive. Both $A \beta$ and Amylin are misfolded peptides. The direct interaction of misfolded peptides, a topic which to date has been poorly explored, could play a major role in the genesis and progression of several pathological conditions. Although not extensively studied, in vitro reports show cross-seeding interactions among several amyloidogenic proteins. ${ }^{14-20}$ One of these studies ${ }^{19}$ showed that $\mathrm{A} \beta_{1-42}$ acts as a good seed for Amylin ${ }_{1-37}$ oligomerization; however, Amylin ${ }_{1-37}$ aggregates slightly affect soluble $A \beta_{1-42}$ oligomerization. A recent study applied electrospray ionization-ion mobility spectroscopy-mass 
spectroscopy to characterize the dynamics and the kinetics of Amylin $_{1-37}$ oligomerization, $A \beta_{1-40}$ oligomerization and Amylin $_{1-37^{-}}$ $\mathrm{A} \beta_{1-40}$ oligomerization. ${ }^{21}$ The interactions between Amylin $_{1-37}$ aggregates and $A \beta_{1-42}$ aggregates at atomic resolution are still elusive.

Several studies proposed that the sequences of $A \beta_{1-42}$ and Amylin $_{1-37}$ have $25 \%$ identity and $50 \%$ similarity and thus some domains in $\mathrm{A} \beta$ and some in Amylin participate in the co-assembly of $\mathrm{A} \beta$-Amylin. ${ }^{22-26}$ Yet, these studies do not provide the atomic resolution of the molecular structures of $A \beta_{1-42}-A m y l i n n_{1-37}$ aggregates. Recently, Berhanu et $a .^{27}$ investigated the molecular structures of $\mathrm{A} \beta_{15-40}-$ Amylin $_{10-35}$ oligomers at atomic resolution. They explored an $A \beta_{15-40}$ oligomer fragment of the ssNMR model of $A \beta_{17-42}{ }^{28}$ not considering the toxic full-length $A \beta_{1-42}$ oligomer, arguing that residues 1-16 in the $\mathrm{N}$-terminus of $\mathrm{A} \beta$ are in a disordered domain and thus unlikely to play a role in aggregation. However, previous studies have shown that residues 1-16 in the N-terminus of $A \beta$ can play important roles in fibrilization and form a well-organized $\beta$-strand structure. ${ }^{29-33}$ It is known that several mutations in the $\mathrm{N}$-terminus accelerate amyloid formation, such as the English (H6R) mutation; ${ }^{34}$ in addition, mutating Ala2 to Thr or Val modifies the $\mathrm{A} \beta$ aggregation landscape. ${ }^{35-38}$ Amylin $_{10-35}$ oligomers of one of the two structures proposed by the Eisenberg group, ${ }^{39}$ which differ in the orientation of the residues along the U-turn region and thus can strongly affect the interaction between $A \beta$ and Amylin, ${ }^{39}$ were explored, ${ }^{27,40}$ but not those proposed by the Tycko group. ${ }^{41}$ Recently, our group illustrated that Amylin ${ }_{1-37}$ oligomers have four variant models that differ in the orientation of the residues along the $\beta$-strands and the turn region of the $\beta$-arch. ${ }^{42}$ The interactions between $\mathrm{A} \beta$ and Amylin may differ due to these different orientations. Although residues 1-7 in the N-terminus of Amylin are not part of the $\beta$-sheet of the experimental models and the disulfide bridge between Cys2 and Cys7 does not contribute to aggregate assembly, it may have an effect on $\mathrm{A} \beta$-Amylin aggregation when considering single and double layer conformations. Residues Ala8 and Thr9 in Amylin that were overlooked in earlier studies ${ }^{27}$ may also contribute to $A \beta$-Amylin aggregation. Finally, the N-terminus of A $\beta$, residues Asp1-Lys16, may interact with Amylin and thus affect cross-seeding of $A \beta$-Amylin aggregation. Here we study the interactions between $A \beta_{1-42}$ oligomers with each of the four models of the full-length Amylin $_{1-37}$ oligomers at atomic resolution. Our results led to two important conclusions. First, all four variant models of the full-length Amylin $_{1-37}$ oligomers prefer to interact with $A \beta_{1-42}$ oligomers to form single layer conformations. Second, interactions between the cross-seeded species in the single layer and the double layer conformations affect differently the flexibility (or rigidity) of the turn region of the self-assembled $\beta$-arch amyloids. Finally, residues in the N-termini of $A \beta$ and Amylin contribute to the cross-seeding $A \beta$-Amylin aggregation; therefore it is important to consider the full-length $A \beta$ and Amylin.

\section{Materials and methods}

\section{Molecular dynamics protocol}

MD simulations of the solvated oligomers were performed in the $N P T$ ensemble using NAMD ${ }^{43}$ with the CHARMM27 force field. ${ }^{44,45}$
The oligomers were energy minimized and explicitly solvated in a TIP3P water box ${ }^{46,47}$ with a minimum distance of $15 \AA$ from each edge of the box. Each water molecule within $2.5 \AA$ of the oligomers was removed. Counter ions were added at random locations to neutralize the charge of the oligomers. The Langevin piston $\operatorname{method}^{43,48,49}$ with a decay period of $100 \mathrm{fs}$ and a damping time of $50 \mathrm{fs}$ was used to maintain a constant pressure of $1 \mathrm{~atm}$. The temperature $(330 \mathrm{~K})$ was controlled by a Langevin thermostat with a damping coefficient of $10 \mathrm{ps}^{43}$ The short-range van der Waals (VDW) interactions were calculated using the switching function, with a twin range cutoff of 10.0 and $12.0 \AA$ A. Long-range electrostatic interactions were calculated using the particle mesh Ewald method with a cutoff of $12.0 \AA^{50,51}$ The equations of motion were integrated using the leapfrog integrator with a step of $1 \mathrm{fs}$.

The solvated systems were energy minimized for 2000 conjugated gradient steps, where the hydrogen bonding distance between the $\beta$-sheets in each oligomer is fixed in the range 2.2-2.5 A. The counter ions and water molecules were allowed to move. Hydrogen atoms were constrained to the equilibrium bond using the SHAKE algorithm. ${ }^{52}$ The minimized solvated systems were energy minimized for 5000 additional conjugate gradient steps and 20000 heating steps at $250 \mathrm{~K}$, with all atoms allowed to move. Then, the systems were heated from $250 \mathrm{~K}$ to $300 \mathrm{~K}$ and then to $330 \mathrm{~K}$ for $300 \mathrm{ps}$ and equilibrated at $330 \mathrm{~K}$ for 300 ps. Simulations ran for $30 \mathrm{~ns}$ for each variant model, with a total run of $960 \mathrm{~ns}$ for all models. The structures were saved every 10 ps for analysis. These conditions were applied to all models.

\section{Generalized Born method with molecular volume (GBMV) and population analysis}

To obtain the relative structural stability of the models, the trajectories of the last $5 \mathrm{~ns}$ were extracted from the explicit MD simulation excluding water molecules. The solvation energies of all systems were calculated using the generalized Born method with molecular volume (GBMV). ${ }^{53,54}$ In the GBMV calculations, the dielectric constant of water was set to 80.0. The hydrophobic solvent-accessible surface area (SASA) term factor was set to $0.00592 \mathrm{kcal} \mathrm{mol}^{-1}$. $^{2}$ Each variant was minimized for 1000 cycles and the conformation energy was evaluated by gridbased GBMV. The minimization does not change the conformations of each variant, but only relaxed the local geometries due to thermal fluctuation which occurred during the MD simulations.

A total of 16000 conformations (500 conformations for each of the 32 examined conformers) were used to construct the free energy landscape of the conformers and to evaluate the conformer probabilities by using Monte Carlo (MC) simulations. In the first step, one conformation of conformer $i$ and one conformation of conformer $j$ were randomly selected. Then, the Boltzmann factor was computed as $\mathrm{e}^{-\left(E_{j}-E_{i}\right)} / k T$, where $E_{i}$ and $E_{j}$ are the conformational energies evaluated using the GBMV calculations for conformations $i$ and $j$, respectively, $k$ is the Boltzmann constant and $T$ is the absolute temperature $(298 \mathrm{~K}$ used here). If the value of the Boltzmann factor was larger than the random number, then the move from conformation $i$ to conformation $j$ was allowed. After 1 million steps, the visited conformations for each conformer were counted. Finally, the 
relative probability of model $\mathrm{n}$ was evaluated as $P_{\mathrm{n}}=N_{\mathrm{n}} / N_{\text {total }}$, where $P_{\mathrm{n}}$ is the population of model $\mathrm{n}, N_{\mathrm{n}}$ is the total number of conformations visited for model $\mathrm{n}$, and $N_{\text {total }}$ is the total steps. The advantages of using MC simulations to estimate conformer probability lie in their good numerical stability and the control that they allow for transition probabilities among several conformers.

Using all 32 models and 16000 conformations (500 for each model) generated from the MD simulations, we estimated the overall stability and populations for each conformer based on the MD simulations, with the energy landscape being computed with GBMV for these 32 models. The group that these 32 models are likely to represent may be only a very small percentage of the ensemble. Nevertheless, the carefully selected models cover the most likely structures. It should be noted here that the results obtained in this study depend on the initial structures and the initial conditions.

\section{Analysis details}

We examined the structural stability of the models by following the changes in the number of the hydrogen bonds between $\beta$-strands, with the hydrogen bond cut-off set to $2.5 \AA$. In addition we followed the root-mean square deviations (RMSDs) and root-mean square fluctuations (RMSFs) of all structures. The $\psi$ and $\varphi$ angles of each residue in the Amylin models were computed for the last $5 \mathrm{~ns}$ to estimate the secondary structure of the self-assembled models.

\section{Reaction coordinates for the formation of Amylin-A $\beta$ oligomer structures}

To investigate the stability of each soluble Amylin-A $\beta$ oligomer structure, the conformational energies were computed for all Amylin oligomers and for the A $\beta$ oligomer (Table S1, ESI $\dagger$ ). The conformational energies for each model are based on the energy computed with the GBMV method. For each model, a total of 500 conformations from the last $5 \mathrm{~ns}$ of the simulations were used to evaluate the conformational energy.

We estimated the relative stability of each Amylin-A $\beta$ model by comparing its energy with the energies of its two types of components, the Amylin model and the $A \beta$ oligomer, as illustrated by the following chemical "reaction":

$$
(\text { Amylin })_{n}+(\mathrm{A} \beta)_{n} \Leftrightarrow(\text { Amylin })_{n} \cdot(\mathrm{A} \beta)_{n}
$$

where $n$ indicates the number of monomers within the Amylins' model and the $\mathrm{A} \beta$ oligomer. In the current study $n=6$.

Obviously, single and double layer models may have different interaction types. Yet, as we previously demonstrated ${ }^{31-33,40,42,55,56}$ one can compare the relative conformational energies between the single and the double layer models to provide insight into these different interactions. The differences in the interaction types may explain the differences in the relative energies.

\section{Results and discussion}

Experiment-based Amylin 1-37 oligomer model, $A \beta_{1-42}$ oligomer model and $A \beta_{1-42}-A_{m y l i n}-37$ oligomer model construction

Previously, we illustrated four models of Amylin 1-37 $_{1 \text { that differ }}$ in the orientation of the residues along the backbone of the $\beta$-strands and along the turn domain of the $\beta$-arch structure. ${ }^{42}$ These four models (M1-M4, Fig. S1, ESI $\dagger$ ) were based on the experimental structures of Tycko ${ }^{41}$ and Eisenberg. ${ }^{39}$ Herein, on the basis of Tycko's two-fold $A \beta_{1-40}$ model, ${ }^{57}$ we extended the C-terminus by two residues to form $A \beta_{1-42}$, which is more toxic than $A \beta_{1-40}$. For each of the four Amylin ${ }_{1-37}$ oligomers we constructed eight models of $A \beta_{1-42}-A m y l i n_{1-37}$ oligomers, where each model consists of six $A \beta_{1-42}$ monomers and six Amylin $_{1-37}$ monomers. Four were single layer conformations and four double layer conformations. Therefore, we constructed a total of $32 \mathrm{~A} \beta_{1-42}-$ Amylin $_{1-37}$ models. Table 1 details all 32 $\mathrm{A} \beta_{1-42}-$ Amylin $_{1-37}$ models: B1-B8, C1-C8 and D1-D8. Fig. 1 illustrates the eight initial models of $A \beta_{1-42}-\mathrm{Amylin}_{1-37}$ oligomers of one of the four Amylin ${ }_{1-37}$ models (model M1): B1-B8. Similarly we constructed the arrangements between $A \beta_{1-42}$ oligomers and Amylin ${ }_{1-37}$ oligomers (models M2, M3 and M4). Fig. S2-S4 (ESI $\dagger$ ) demonstrate the other 24 initial $A \beta_{1-42}-\mathrm{Amylin}_{1-37}$ models and Fig. S5-S8 show the 32 simulated $A \beta_{1-42}-$ Amylin $_{1-37}$ models. Finally, we simulated the $A \beta_{1-42}$ oligomer model which is based on Tycko's two-fold $A \beta_{1-40}$ model (Fig. S9a, ESI $\dagger$ ). ${ }^{57}$ Interestingly, the simulated $A \beta_{1-42}$ oligomer model illustrated a

Table 1 The investigated 32 models of $A \beta_{1-42}-A m y l i n_{1-37}$ dodecamers: $A \beta_{1-42}$ hexamers are based on Tycko's structure (ref. 51 ) and the four types of Amylin ${ }_{1-37}$ hexamers M1-M4 were taken from Miller's structures (ref. 36) [based on Tycko's ssNMR (ref. 35) and Eisenberg's crystal structures (ref. 34)]. $A \beta_{1-42}$ hexamers and Amylin 1-37 $_{1}$ hexamers in models B3, C3, D3 and E3 are constructed in an antiparallel orientation. $A \beta_{1-42}$ hexamers and Amylin $1-37$ hexamers in models B4, C4, D4 and E4 are constructed in parallel dimers that are organized in an antiparallel orientation

\begin{tabular}{|c|c|c|}
\hline Model & Amylin type & $\begin{array}{l}\text { Orientations between } \\
\mathrm{A} \beta \text { and Amylin }\end{array}$ \\
\hline B1 & M1 & Parallel \\
\hline B2 & M1 & Antiparallel \\
\hline B3 & M1 & Parallel \\
\hline B4 & M1 & Antiparallel \\
\hline B5 & M1 & $\mathrm{N}(\mathrm{A} \beta)-\mathrm{N}($ Amylin) \\
\hline B6 & M1 & $\mathrm{N}(\mathrm{A} \beta)-\mathrm{C}($ Amylin $)$ \\
\hline B7 & M1 & $\mathrm{C}(\mathrm{A} \beta)-\mathrm{N}($ Amylin) \\
\hline B8 & M1 & $\mathrm{C}($ Amylin $)-\mathrm{C}(\mathrm{A} \beta)$ \\
\hline $\mathrm{C} 1$ & M2 & Parallel \\
\hline $\mathrm{C} 2$ & M2 & Antiparallel \\
\hline C3 & M2 & Parallel \\
\hline $\mathrm{C} 4$ & M2 & Antiparallel \\
\hline C5 & M2 & $\mathrm{N}(\mathrm{A} \beta)-\mathrm{N}($ Amylin) \\
\hline C6 & M2 & $\mathrm{N}(\mathrm{A} \beta)-\mathrm{C}($ Amylin) \\
\hline C7 & M2 & $\mathrm{C}(\mathrm{A} \beta)-\mathrm{N}($ Amylin) \\
\hline $\mathrm{C} 8$ & M2 & $\mathrm{C}($ Amylin) $-\mathrm{C}(\mathrm{A} \beta)$ \\
\hline D1 & M3 & Parallel \\
\hline D2 & M3 & Antiparallel \\
\hline D3 & M3 & Parallel \\
\hline D4 & M3 & Antiparallel \\
\hline D5 & M3 & $\mathrm{N}(\mathrm{A} \beta)-\mathrm{N}($ Amylin) \\
\hline D6 & M3 & $\mathrm{N}(\mathrm{A} \beta)-\mathrm{C}($ Amylin $)$ \\
\hline D7 & M3 & $\mathrm{C}(\mathrm{A} \beta)-\mathrm{N}($ Amylin) \\
\hline D8 & M3 & $\mathrm{C}($ Amylin $)-\mathrm{C}(\mathrm{A} \beta)$ \\
\hline E1 & M4 & Parallel \\
\hline $\mathrm{E} 2$ & M4 & Antiparallel \\
\hline E3 & M4 & Parallel \\
\hline $\mathrm{E} 4$ & M4 & Antiparallel \\
\hline E5 & M4 & $\mathrm{N}(\mathrm{A} \beta)-\mathrm{N}($ Amylin) \\
\hline E6 & M4 & $\mathrm{N}(\mathrm{A} \beta)-\mathrm{C}($ Amylin $)$ \\
\hline E7 & M4 & $\mathrm{C}(\mathrm{A} \beta)-\mathrm{N}($ Amylin) \\
\hline E8 & M4 & $\mathrm{C}($ Amylin $)-\mathrm{C}(\mathrm{A} \beta)$ \\
\hline
\end{tabular}



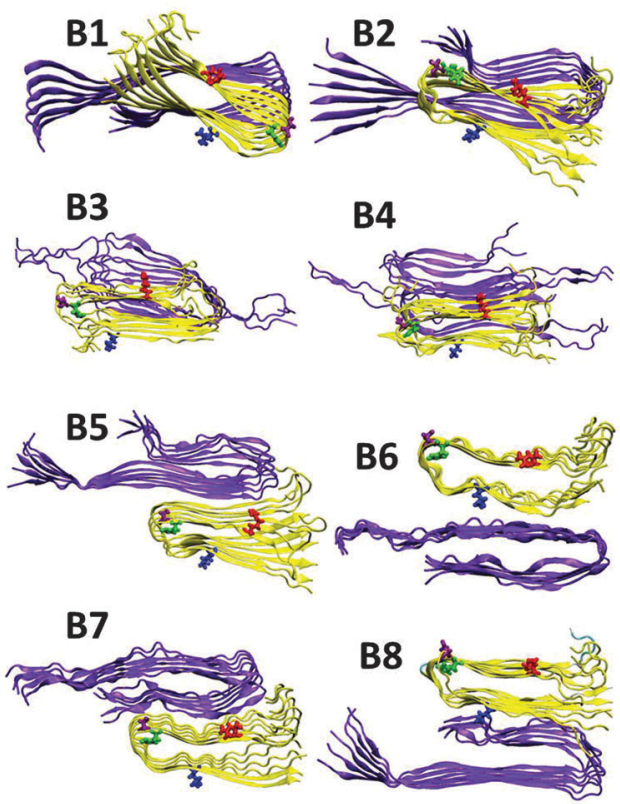

Fig. 1 Constructed initial $A \beta_{1-42}-$ Amylin $_{1-37}$ dodecamers. $A \beta$ hexamer is based on Tycko's structure (ref. 51) and the Amylin hexamer M1 is obtained from Miller's structures (ref. 36) [based on Tycko's ssNMR (ref. 35) and Eisenberg's crystal structures (ref. 34)]. Models B1-B4 are single layer conformations and B5-B8 are double layer conformations.

third $\beta$-stand at the C-termini of the monomers (Fig. S9b, $\mathrm{ESI}^{\dagger}$ ) leading to a new structural model of the $A \beta_{1-42}$ oligomer. Recently, a novel structural model of the $A \beta_{11-42}$ oligomer provided further evidence for the highly polymorphic nature of the $A \beta$ peptide fibril. ${ }^{58}$ Polymorphism was also obtained in some of the simulated $A \beta_{1-42}-$ Amylin $_{1-37}$ models. Structural comparison suggests that the structural similarity between the $A \beta_{11-42}$ oligomer and amylin oligomers is lower than with the model of the $A \beta$ oligomer in the current study. It will be interesting to examine the cross-seeding $\mathrm{A} \beta_{1-42}-$ Amylin $_{1-37}$ oligomers using this new $\mathrm{A} \beta$ structure $^{59}$ as well as with additional polymorphic states such as the triangular structure. ${ }^{60}$ Previous SSNMR studies have shown $\alpha$-helical structures of Amylin and not cross- $\beta$ structures. ${ }^{61,62}$ Recent sSNMR studies presented unstructured $A \beta$ oligomers ${ }^{63}$ and Amylin oligomers that form large micelles, ${ }^{64}$ which may be a general phenomenon for natively unstructured Amylin. We did not apply these $\alpha$-helical and the unstructured amylin, or the unstructured $A \beta$ oligomers in the current study, because of the lack of the PDB coordinates. Future work would need to solve these oligomer structures in order to study the cross-seeding of $A \beta_{1-42}-$ Amylin $_{1-37}$ oligomers. Finally, the RMSDs and the hydrogen bond analysis illustrate that the simulated $A \beta_{1-42}-\mathrm{Amylin}_{1-37}$ models are structurally stable (Fig. S10-S15, ESI $\dagger$ ).

Single layer conformational arrangements of $\mathbf{A} \boldsymbol{\beta}_{1-42}-\mathbf{A m y l i n}{ }_{1-37}$ oligomer models are preferred over double layer conformational arrangements

In order to compare the $32 A \beta_{1-42}-A_{m y l i n}{ }_{1-37}$ oligomers we generated 500 conformations for each arrangement by MD simulations and estimated the conformational energies and (a)

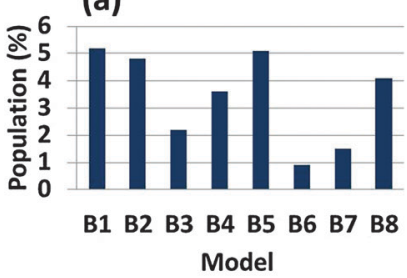

(c)

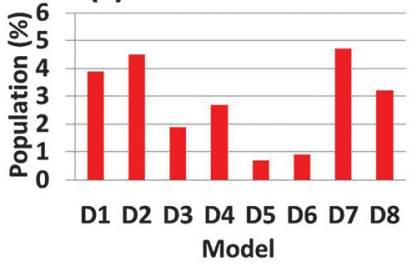

(d)

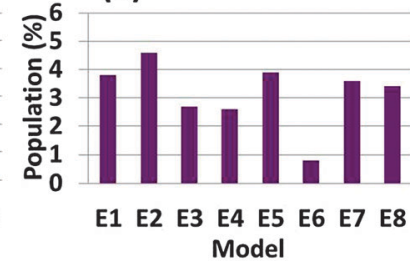

Fig. 2 Populations of the simulated $A \beta_{1-42}-$ Amylin $_{1-37}$ dodecamers, which are estimated by Monte-Carlo simulations.

the populations (Table S1, ESI $\dagger$ ). Fig. 2 demonstrates the populations of all $32 \mathrm{~A} \beta_{1-42}-$ Amylin $_{1-37}$ oligomers. One can see that single layer conformations of $A \beta_{1-42}-$ Amylin $_{1-37}$ oligomers with parallel and antiparallel arrangements (B1, B2, C1, C2, D1, D2, E1 and E2) show the highest populations and thus are preferred over the two single layer conformations and all the double layer conformations. We previously showed that in the cross-seeded $\mathrm{A} \beta_{17-42}$-mutated Tau R2 oligomers the double layer conformations are preferred over the single layer conformations. ${ }^{56}$ The preference of the cross-seeding of some conformations over others may be due to the interactions between residues along the sequences of the various types of amyloids. The interactions that stabilize structurally and energetically the cross-seeding amyloid oligomers will yield preferred organizations.

We computed the secondary structures of $A \beta_{1-42}$ oligomers in all 32 cross-seeding Amylin ${ }_{1-37}-\mathrm{A} \beta_{1-42}$ oligomers (Fig. S16-S23, ESI $\dagger$ ). Interestingly, one can see from Fig. S16-S21 (ESI $\dagger$ ) that in the single layer simulated $A \beta_{1-42}-$ Amylin $_{1-37}$ oligomer models and in the double layer simulated $A \beta_{1-42}-$ Amylin $_{1-37}$ oligomer models (in which the C-termini of $A \beta_{1-42}$ monomers do not interact with Amylin) residues Val39-Ala42 in the C-termini of $A \beta_{1-42}$ showed formation of a third $\beta$-strand and a second turn region (residues Gly37-Gly38). The original experiment-based $A \beta_{1-42}$ oligomer $^{57}$ has two $\beta$-strands connected by a U-turn; however, herein our simulations demonstrate that the $A \beta_{1-42}$ oligomer has three $\beta$-strands connected by two turns both when it does not interact with Amylin $_{1-37}$ oligomers and when it does. A previous study has shown that isoforms of Tau repeats form triple-stranded and two-turn structures. ${ }^{65}$

\section{Common mechanisms between various types of Amylin 1-37} oligomers and $\mathbf{A} \boldsymbol{\beta}_{1-42}$ oligomers

To investigate the mechanisms by which Amylin $_{1-37}$ oligomers interact with $A \beta_{1-42}$ oligomers to form the cross-seeded Amylin $_{1-37}-\mathrm{A} \beta_{1-42}$ oligomers, we estimated the "reaction coordinate" in which Amylin ${ }_{1-37}$ oligomers interact with $A \beta_{1-42}$ oligomers. To this aim, we computed the relative conformational energies of the separated oligomers and the cross-seeding Amylin ${ }_{1-37}-\mathrm{A} \beta_{1-42}$ 
oligomers using the GBMV method $^{53,54}$ for each of the four Amylin $_{1-37}$ oligomers (Fig. 3). ${ }^{42}$ We previously studied similarly the cross-seeding interactions between two types of amyloids ${ }^{55,56}$ and two types of Amylin ${ }_{1-37}{ }^{42}$

Interestingly, the common mechanisms by which Amylin 1-37 oligomers interact with $A \beta_{1-42}$ oligomers to form the cross-seeded Amylin $_{1-37}-\mathrm{A} \beta_{1-42}$ oligomers illustrate that each of the four Amylin $_{1-37}$ oligomers prefers to interact with an $A \beta_{1-42}$ oligomer to form a single layer conformation in parallel and in antiparallel orientation, yielding an 'exothermic reaction'. In some of the four Amylin $_{1-37}$ oligomers there are other mechanisms that illustrate the formation of double layer conformations, but in all four Amylin $_{1-37}$ oligomers the common mechanisms show the formation of the single layer conformation. Therefore, the preference of the single layer conformation indicates a strong cross-seeding tendency between $\mathrm{A} \beta_{1-42}$ and Amylin $_{1-37}$ peptides.

\section{The effect of cross-seeding on the structural features of} Amylin $_{1-37}$ oligomers and $\mathbf{A} \boldsymbol{\beta}_{1-42}$ oligomers

One of the interesting topics in studying the cross-seeding between amyloids is to investigate the effect of cross-seeding on the structural features of the amyloids. The cross-seeding between Amylin $_{1-37}$ oligomers and $A \beta_{1-42}$ oligomers is of particular interest, because there are four variant models of Amylin ${ }_{1-37}$ oligomers that differ in the orientation of the residues along the $\beta$-arch structures and thus we expect that the effect on the structural features of the various cross-seeding Amylin $_{1-37}-\mathrm{A} \beta_{1-42}$ oligomers may be different.

We first examined the effect on the $\beta$-strand of the $\beta$-arch structures of $A \beta_{1-42}$ oligomers. The secondary structures of these oligomers in all 32 cross-seeding Amylin ${ }_{1-37}-A \beta_{1-42}$ oligomers were computed (Fig. S16-S23, ESI $\dagger$ ). In $\mathrm{A} \beta_{1-42}$ oligomers residues D1-F20 and residues A30-A42 show the properties of $\beta$-strand, but in the cases (models B6, C6, D6 and E6) where the N-termini of $A \beta_{1-42}$ oligomers interact with the C-termini of Amylin $_{1-37}$ oligomers to form double layer conformations these residues do not demonstrate $\beta$-strand properties. Also, in some cases (models C5 and D5) where the N-termini of $A \beta_{1-42}$ oligomers interact with the $\mathrm{N}$-termini of Amylin $_{1-37}$ oligomers or in one case (model B7) where the C-termini of $A \beta_{1-42}$ oligomers interact with the $\mathrm{N}$-termini of Amylin $_{1-37}$ oligomers these residues do not show $\beta$-strand properties. In such cases the cross- $\beta$ structures that characterize the fibrillation of amyloids yield structurally less stable cross-seeding.

We then examined the fluctuation of the backbone of $\mathrm{A} \beta_{1-42}$ oligomers and Amylin ${ }_{1-37}$ oligomers for each of the 32 crossseeding Amylin ${ }_{1-37}-\mathrm{A} \beta_{1-42}$ oligomers using RMSF calculations (Fig. 4 and 5). Interestingly, in cases (models B6, C6, D6 and E6) where the N-termini of $A \beta_{1-42}$ oligomers interact with the C-termini of Amylin ${ }_{1-37}$ oligomers to form double layer conformations, the turn regions of $\mathrm{A} \beta_{1-42}$ oligomers fluctuate relatively more than in other cross-seeding models. These models demonstrated no $\beta$-strand properties, because the interactions in the double layer conformations do not allow structurally stable structures. One can see from the simulated models (Fig. S5-S8, ESI $\dagger$ ) that $A \beta_{1-42}$ oligomers do not show cross- $\beta$ structures, and are structurally unstable oligomers. Finally, those simulated models that do not exhibit cross- $\beta$ structures (models C5, D5 and B7) also fluctuate in the turn region of the $A \beta_{1-42}$ oligomers.

Our results suggest that among the double layer conformations of the cross-seeding oligomers, the stability of the turn region domain in the $A \beta_{1-42}$ oligomers may be affected by the
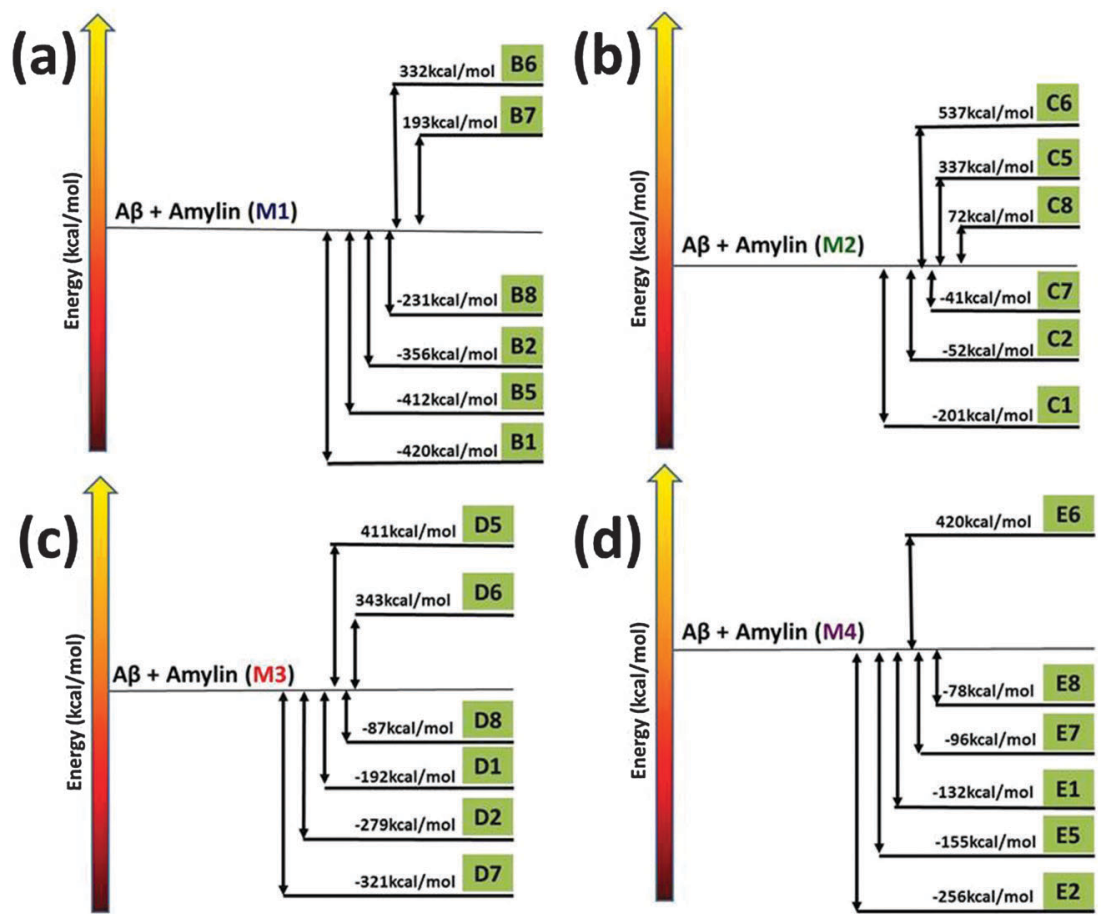

Fig. 3 The relative conformational energies of separated $A \beta_{1-42}$ hexamers and Amylin ${ }_{1-37}$ hexamers (M1-M4) and $A \beta_{1-42}-A m y l i n_{1-37}$ dodecamers. 


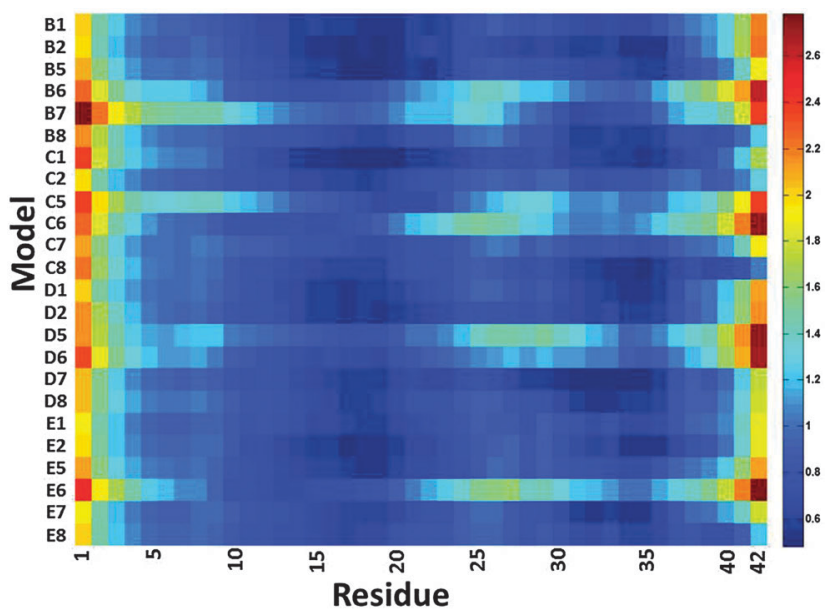

Fig. 4 The averaged RMSF of residues of $A \beta_{1-42}$ in the 32 models of $\mathrm{A} \beta_{1-42}-$ Amylin $_{1-37}$ dodecamers.

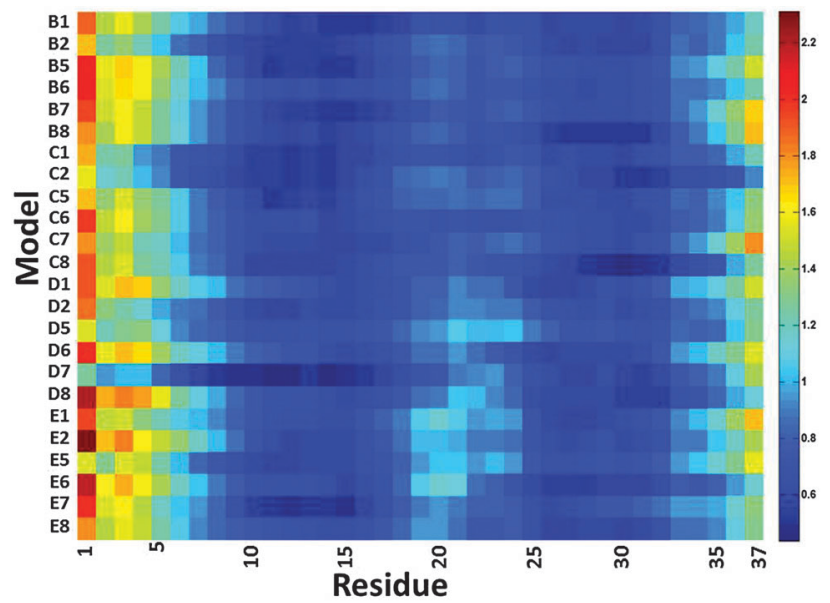

Fig. 5 The averaged RMSF of residues of Amylin $_{1-37}$ in the 32 models of $A \beta_{1-42}-$ Amylin $_{1-37}$ dodecamers.

interactions with all four Amylin ${ }_{1-37}$ oligomer variants suggesting that the turn region may affect the fibrillation (or the selfassembly process) of $A \beta_{1-42}$ oligomers. In cases where the turn regions in $A \beta_{1-42}$ oligomers are destabilized due to the interactions with Amylin ${ }_{1-37}$ oligomers, these interactions may inhibit aggregation of the $A \beta_{1-42}$ oligomers. Zanni's group proposed an aggregation pathway for amylin in which the turn regions of amylin play a role as initial seeding for aggregation. ${ }^{66}$ We thus suggest that in some cases destabilization of the turn regions of $A \beta_{1-42}$ oligomers may inhibit $A \beta_{1-42}$ aggregation. However, in some cases the turn regions in $A \beta_{1-42}$ oligomers are stabilized by these interactions and therefore we expect that these interactions will induce aggregation of $A \beta_{1-42}$ oligomers.

Our study illustrates for the first time the importance of investigating the cross-seeding between full-length $\mathrm{A} \beta$ and full-length Amylin and that the $\mathrm{N}$-termini play a role in some cases in the stabilization of the cross-seeding of $A \beta$-Amylin oligomers. One can see that interactions between residues in the N-termini of $A \beta$ and Amylin (those that had not been considered earlier ${ }^{27}$ ) stabilize both single and double layer conformations (Fig. S24-S26, ESI $\dagger$ ). On the other hand, interestingly, in some cases the interactions between the Val12 of A $\beta$ (which is located in the $\mathrm{N}$-terminus) and the C-terminus of Amylin (residues Ile26 and Leu27) destabilize the A $\beta$ oligomers but do not affect the stabilization of Amylin (Fig. S27, ESI $\dagger$ ). Therefore, this is the first study that illustrates the role of the interactions of the $\mathrm{N}$-termini in cross-seeding $\mathrm{A} \beta$-Amylin aggregation at atomic resolution. In some cases, the $\mathrm{N}$-termini are favorable for cross-seeding and in some other cases the $\mathrm{N}$-termini are unfavorable for cross-seeding.

Finally, we examined the effect of the interactions of $A \beta_{1-42}$ oligomers on the fluctuation of the turn regions of Amylin ${ }_{1-37}$ oligomers. Interestingly, one can see from Fig. 5 that the interactions of $A \beta_{1-42}$ oligomers with the variant models of Amylin $_{1-37}$ oligomers (M3 and M4) result in fluctuations of the turn regions of Amylin $_{1-37}$ oligomers, i.e. flexibility of the turn regions, which is in contrast with the interactions of $A \beta_{1-42}$ oligomers with the variant models of Amylin ${ }_{1-37}$ oligomers (M1 and M2) which result in more rigid turn regions. Previously we showed that the variant models M1 and M2 of Amylin ${ }_{1-37}$ oligomers illustrated rigid turn regions, while with the variant

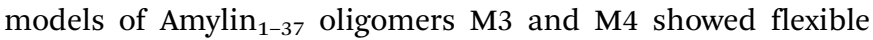
turn regions. ${ }^{42}$ We thus suggest that the interactions of $A \beta_{1-42}$ oligomers do not affect the structural features of Amylin ${ }_{1-37}$ oligomers; rather, in some cases Amylin ${ }_{1-37}$ oligomers affect the structural features and the stability of $A \beta_{1-42}$ oligomers.

\section{Conclusions}

Many, though not all, clinical studies indicate that individuals with T2D are at higher risk of eventually developing AD or other dementia, ${ }^{1-3}$ but the connection between these two diseases is not understood. Recently, Amylin deposits were found in the temporal lobe gray matter - a major component of the central nervous system - of diabetes patients. ${ }^{10}$ In addition to the Amylin deposition in the human brain, Amylin aggregates were found to co-localize with $\mathrm{A} \beta$ aggregates to form Amylin-A $\beta$ plaques, promoting aggregation and thus contributing to the etiology of AD. The mechanisms by which Amylin co-aggregates with $A \beta$ are still elusive. Herein, we present for the first time the co-aggregation between the full-length $A \beta_{1-42}$ oligomer and each of the four variants of the full-length Amylin ${ }_{1-37}$ oligomers that we have recently established. ${ }^{42}$ Here, we focus on the parallel $\beta$-sheet structure of the $A \beta_{1-42}$ oligomer, although the antiparallel $\beta$-sheet structure of $\mathrm{A} \beta_{1-40}$ oligomers has also been considered as a toxic species. ${ }^{67}$ Yet, it is known that $A \beta_{1-42}$ is more toxic than $A \beta_{1-40}$. The toxicity of $A \beta$ oligomers to neuronal cells has been demonstrated to occur via a two-step mechanism of membrane disruption. ${ }^{68}$

Two important observations emerge from this study. First, all four variant models of the full-length Amylin $_{1-37}$ oligomers preferred to interact with $\mathrm{A} \beta_{1-42}$ oligomers to form polymorphic single layer conformations. Second, the interactions between the cross-seeding Amylin ${ }_{1-37}-\mathrm{A} \beta_{1-42}$ oligomers both in single 
and in double layer conformations affect the structural features differently. In particular, the differences center on the flexibility/ rigidity of the turn region and the order/disorder of the $\beta$-strands in the self-assembled $\beta$-arch amyloids. Finally, while studying cross-seeding Amylin $_{1-37}-\mathrm{A} \beta_{1-42}$ oligomers, it is important to investigate the full-length of these amyloids because of the role that the terminal residues may play in the stabilization of the hetero-oligomers. Understanding the mechanisms and the range of structural features of the co-aggregates of Amylin $_{1-37}-\mathrm{A} \beta_{1-42}$ oligomers is of crucial importance for effective drug design to reduce co-aggregate formation and maybe to prevent patients with $\mathrm{T} 2 \mathrm{D}$ from developing $\mathrm{AD}$ in later life. A recent study demonstrated the cross-seeding effects of bacterial curli on semen enhancer of viral infection (SEVI), A $\beta$ and Amylin. ${ }^{69}$ This experimental study reports important implications and it would be useful to further strengthen this study using MD simulations, as reported in the present study.

\section{Acknowledgements}

This project was supported by the FP7-PEOPLE-2011-CIG, research grant no. 303741, and in whole or in part by Federal funds from the National Cancer Institute, National Institutes of Health, under contract number HHSN261200800001E. This research was supported (in part) by the Intramural Research Program of the $\mathrm{NIH}$, National Cancer Institute, Center for Cancer Research.

All simulations were performed using the high-performance computational facilities of the Miller Lab in the BGU HPC computational center and the Biowulf PC/Linux cluster at the National Institutes of Health, Bethesda, MD (http://biowulf.nih. gov). The support of the BGU HPC computational center staff is greatly appreciated.

\section{References}

1 J. Gotz, L. M. Ittner and Y. A. Lim, Common features between diabetes mellitus and Alzheimer's disease, Cell. Mol. Life Sci., 2009, 66, 1321-1325.

2 Y. Yang and W. Song, Molecular links between Alzheimer's disease and diabetes mellitus, Neuroscience, 2013, 250, 140-150.

3 L. Li and C. Holscher, Common pathological processes in Alzheimer disease and type 2 diabetes: a review, Brain Res. Rev., 2007, 56, 384-402.

4 I. Churcher, Tau therapeutic strategies for the treatment of Alzheimer's disease, Curr. Top. Med. Chem., 2006, 6, 579-595.

5 C. G. Glabe, Common mechanisms of amyloid oligomer pathogenesis in degenerative disease, Neurobiol. Aging, 2006, 27, 570-575.

6 J. Gotz, Y. A. Lim and A. Eckert, Lessons from two prevalent amyloidoses - what amylin and Abeta have in common, Front. Aging Neurosci., 2013, 5, 38.

7 A. M. Brands, R. P. Kessels, E. H. de Haan, L. J. Kappelle and G. J. Biessels, Cerebral dysfunction in type 1 diabetes: effects of insulin, vascular risk factors and blood-glucose levels, Eur. J. Pharmacol., 2004, 490, 159-168.
8 M. Ristow, Neurodegenerative disorders associated with diabetes mellitus, J. Mol. Med., 2004, 82, 510-529.

9 E. Roche, J. A. Reig, A. Campos, B. Paredes, J. R. Isaac, S. Lim, R. Y. Calne and B. Soria, Insulin-secreting cells derived from stem cells: clinical perspectives, hypes and hopes, Transplant Immunol., 2005, 15, 113-129.

10 K. Jackson, G. A. Barisone, E. Diaz, L. W. Jin, C. DeCarli and F. Despa, Amylin deposition in the brain: a second amyloid in Alzheimer disease?, Ann. Neurol., 2013, 74, 517-526.

11 M. E. Oskarsson, J. F. Paulsson, S. W. Schultz, M. Ingelsson, P. Westermark and G. T. Westermark, In vivo seeding and cross-seeding of localized amyloidosis: a molecular link between type 2 diabetes and Alzheimer disease, Am. J. Pathol., 2015, 185, 834-846.

12 L. M. Yan, A. Velkova and A. Kapurniotu, Molecular characterization of the hetero-assembly of beta-amyloid peptide with islet amyloid polypeptide, Curr. Pharm. Des., 2014, 20, 1182-1191.

13 W. Q. Qiu, M. Wallack, M. Dean, E. Liebson, M. Mwamburi and $\mathrm{H}$. Zhu, Association between amylin and amyloid-beta peptides in plasma in the context of apolipoprotein E4 allele, PLoS One, 2014, 9, e88063.

14 L. K. Clinton, M. Blurton-Jones, K. Myczek, J. Q. Trojanowski and F. M. LaFerla, Synergistic interactions between Abeta, tau, and alpha-synuclein: acceleration of neuropathology and cognitive decline, J. Neurosci., 2010, 30, 7281-7289.

15 J. P. Guo, T. Arai, J. Miklossy and P. L. McGeer, Abeta and tau form soluble complexes that may promote self aggregation of both into the insoluble forms observed in Alzheimer's disease, Proc. Natl. Acad. Sci. U. S. A., 2006, 103, 1953-1958.

16 M. R. Krebs, L. A. Morozova-Roche, K. Daniel, C. V. Robinson and C. M. Dobson, Observation of sequence specificity in the seeding of protein amyloid fibrils, Protein Sci., 2004, 13, 1933-1938.

17 P. K. Mandal, J. W. Pettegrew, E. Masliah, R. L. Hamilton and R. Mandal, Interaction between Abeta peptide and alpha synuclein: molecular mechanisms in overlapping pathology of Alzheimer's and Parkinson's in dementia with Lewy body disease, Neurochem. Res., 2006, 31, 1153-1162.

18 R. Morales, L. D. Estrada, R. Diaz-Espinoza, D. MoralesScheihing, M. C. Jara, J. Castilla and C. Soto, Molecular cross talk between misfolded proteins in animal models of Alzheimer's and prion diseases, J. Neurosci., 2010, 30, 4528-4535.

19 B. O'Nuallain, A. D. Williams, P. Westermark and R. Wetzel, Seeding specificity in amyloid growth induced by heterologous fibrils, J. Biol. Chem., 2004, 279, 17490-17499.

20 I. F. Tsigelny, L. Crews, P. Desplats, G. M. Shaked, Y. Sharikov, H. Mizuno, B. Spencer, E. Rockenstein, M. Trejo, O. Platoshyn, J. X. Yuan and E. Masliah, Mechanisms of hybrid oligomer formation in the pathogenesis of combined Alzheimer's and Parkinson's diseases, PLoS One, 2008, 3, e3135.

21 L. M. Young, R. A. Mahood, J. C. Saunders, L. H. Tu, D. P. Raleigh, S. E. Radford and A. E. Ashcroft, Insights into the consequences of co-polymerisation in the early stages of IAPP and Abeta peptide assembly from mass spectrometry, Analyst, 2015, DOI: 10.1039/C5AN00865D. 
22 M. A. Schroer, Y. Zhai, D. C. Wieland, C. J. Sahle, J. Nase, M. Paulus, M. Tolan and R. Winter, Exploring the piezophilic behavior of natural cosolvent mixtures, Angew. Chem., 2011, 50, 11413-11416.

23 W. A. Banks, A. J. Kastin, L. M. Maness, W. Huang and J. B. Jaspan, Permeability of the blood-brain barrier to amylin, Life Sci., 1995, 57, 1993-2001.

24 N. Ida, T. Hartmann, J. Pantel, J. Schroder, R. Zerfass, H. Forstl, R. Sandbrink, C. L. Masters and K. Beyreuther, Analysis of heterogeneous A4 peptides in human cerebrospinal fluid and blood by a newly developed sensitive Western blot assay, J. Biol. Chem., 1996, 271, 22908-22914.

25 T. Sanke, T. Hanabusa, Y. Nakano, C. Oki, K. Okai, S. Nishimura, M. Kondo and K. Nanjo, Plasma islet amyloid polypeptide (Amylin) levels and their responses to oral glucose in type 2 (non-insulin-dependent) diabetic patients, Diabetologia, 1991, 34, 129-132.

26 L. M. Yan, M. Tatarek-Nossol, A. Velkova, A. Kazantzis and A. Kapurniotu, Design of a mimic of nonamyloidogenic and bioactive human islet amyloid polypeptide (IAPP) as nanomolar affinity inhibitor of IAPP cytotoxic fibrillogenesis, Proc. Natl. Acad. Sci. U. S. A., 2006, 103, 2046-2051.

27 W. M. Berhanu, F. Yasar and U. H. Hansmann, In silico cross seeding of Abeta and amylin fibril-like oligomers, ACS Chem. Neurosci., 2013, 4, 1488-1500.

28 T. Luhrs, C. Ritter, M. Adrian, D. Riek-Loher, B. Bohrmann, H. Dobeli, D. Schubert and R. Riek, 3D structure of Alzheimer's amyloid-beta(1-42) fibrils, Proc. Natl. Acad. Sci. U. S. A., 2005, 102, 17342-17347.

29 Y. Miller, B. Ma, C. J. Tsai and R. Nussinov, Hollow core of Alzheimer's Abeta42 amyloid observed by cryoEM is relevant at physiological pH, Proc. Natl. Acad. Sci. U. S. A., 2010, 107, 14128-14133.

30 Y. Miller, B. Ma and R. Nussinov, Polymorphism in Alzheimer Abeta amyloid organization reflects conformational selection in a rugged energy landscape, Chem. Rev., 2010, 110, 4820-4838.

31 B. Ma and R. Nussinov, Polymorphic triple beta-sheet structures contribute to amide hydrogen/deuterium (H/D) exchange protection in the Alzheimer amyloid beta42 peptide, J. Biol. Chem., 2011, 286, 34244-34253.

32 B. Ma and R. Nussinov, Polymorphic C-terminal beta-sheet interactions determine the formation of fibril or amyloid beta-derived diffusible ligand-like globulomer for the Alzheimer Abeta42 dodecamer, J. Biol. Chem., 2010, 285, 37102-37110.

33 L. Xu, S. Shan, Y. Chen, X. Wang, R. Nussinov and B. Ma, Coupling of Zinc-Binding and Secondary Structure in Nonfibrillar Abeta40 Peptide Oligomerization, J. Chem. Inf. Model., 2015, 55, 1218-1230.

34 Y. Hori, T. Hashimoto, Y. Wakutani, K. Urakami, K. Nakashima, M. M. Condron, S. Tsubuki, T. C. Saido, D. B. Teplow and T. Iwatsubo, The Tottori (D7N) and English (H6R) familial Alzheimer disease mutations accelerate Abeta fibril formation without increasing protofibril formation, J. Biol. Chem., 2007, 282, 4916-4923.
35 M. Messa, L. Colombo, E. del Favero, L. Cantu, T. Stoilova, A. Cagnotto, A. Rossi, M. Morbin, G. Di Fede, F. Tagliavini and M. Salmona, The peculiar role of the A2V mutation in amyloid-beta (Abeta) 1-42 molecular assembly, J. Biol. Chem., 2014, 289, 24143-24152.

36 I. Benilova, R. Gallardo, A. A. Ungureanu, V. Castillo Cano, A. Snellinx, M. Ramakers, C. Bartic, F. Rousseau, J. Schymkowitz and B. De Strooper, The Alzheimer disease protective mutation A2T modulates kinetic and thermodynamic properties of amyloid-beta (Abeta) aggregation, J. Biol. Chem., 2014, 289, 30977-30989.

37 P. Das, B. Murray and G. Belfort, Alzheimer's protective A2T mutation changes the conformational landscape of the $\operatorname{Abeta}(1)(-)(4)(2)$ monomer differently than does the A2V mutation, Biophys. J., 2015, 108, 738-747.

38 P. H. Nguyen, B. Tarus and P. Derreumaux, Familial Alzheimer A2 $\mathrm{V}$ mutation reduces the intrinsic disorder and completely changes the free energy landscape of the Abeta1-28 monomer, J. Phys. Chem. B, 2014, 118, 501-510.

39 J. J. Wiltzius, S. A. Sievers, M. R. Sawaya, D. Cascio, D. Popov, C. Riekel and D. Eisenberg, Atomic structure of the cross-beta spine of islet amyloid polypeptide (amylin), Protein Sci., 2008, 17, 1467-1474.

40 R. Qi, Y. Luo, B. Ma, R. Nussinov and G. Wei, Conformational distribution and alpha-helix to beta-sheet transition of human amylin fragment dimer, Biomacromolecules, 2014, 15, 122-131.

41 S. Luca, W. M. Yau, R. Leapman and R. Tycko, Peptide conformation and supramolecular organization in amylin fibrils: constraints from solid-state NMR, Biochemistry, 2007, 46, 13505-13522.

42 V. Wineman-Fisher, Y. Atsmon-Raz and Y. Miller, Orientations of residues along the beta-arch of self-assembled amylin fibrillike structures lead to polymorphism, Biomacromolecules, 2015, 16, 156-165.

43 L. Kale, R. Skeel, M. Bhandarkar, R. Brunner, A. Gursoy, N. Krawetz, J. Phillips, A. Shinozaki, K. Varadarajan and K. Schulten, NAMD2: greater scalability for parallel molecular dynamics, J. Comput. Phys., 1999, 151, 283-312.

44 B. R. Brooks, R. E. Bruccoleri, B. D. Olafson, D. J. States, S. Swaminathan and M. Karplus, Charmm - a Program for Macromolecular Energy, Minimization, and Dynamics Calculations, J. Comput. Chem., 1983, 4, 187-217.

45 A. D. MacKerell, D. Bashford, M. Bellott, R. L. Dunbrack, J. D. Evanseck, M. J. Field, S. Fischer, J. Gao, H. Guo, S. Ha, D. Joseph-McCarthy, L. Kuchnir, K. Kuczera, F. T. K. Lau, C. Mattos, S. Michnick, T. Ngo, D. T. Nguyen, B. Prodhom, W. E. Reiher, B. Roux, M. Schlenkrich, J. C. Smith, R. Stote, J. Straub, M. Watanabe, J. Wiorkiewicz-Kuczera, D. Yin and M. Karplus, All-atom empirical potential for molecular modeling and dynamics studies of proteins, J. Phys. Chem. B, 1998, 102, 3586-3616.

46 W. L. Jorgensen, J. Chandrasekhar, J. D. Madura, R. W. Impey and M. L. Klein, Comparison of Simple Potential Functions for Simulating Liquid Water, J. Chem. Phys., 1983, 79, 926-935. 
47 M. W. Mahoney and W. L. Jorgensen, A five-site model for liquid water and the reproduction of the density anomaly by rigid, nonpolarizable potential functions, J. Chem. Phys., 2000, 112, 8910-8922.

48 S. E. Feller, Y. H. Zhang, R. W. Pastor and B. R. Brooks, ConstantPressure Molecular-Dynamics Simulation - the Langevin Piston Method, J. Chem. Phys., 1995, 103, 4613-4621.

49 K. Tu, D. J. Tobias and M. L. Klein, Constant pressure and temperature molecular dynamics simulation of a fully hydrated liquid crystal phase dipalmitoylphosphatidylcholine bilayer, Biophys. J., 1995, 69, 2558-2562.

50 T. Darden, D. York and L. Pedersen, Particle Mesh Ewald an $N \cdot \log (N)$ Method for Ewald Sums in Large Systems, J. Chem. Phys., 1993, 98, 10089-10092.

51 U. Essmann, L. Perera, M. L. Berkowitz, T. Darden, H. Lee and L. G. Pedersen, A Smooth Particle Mesh Ewald Method, J. Chem. Phys., 1995, 103, 8577-8593.

52 J. P. Ryckaert, G. Ciccotti and H. J. C. Berendsen, NumericalIntegration of Cartesian Equations of Motion of a System with Constraints - Molecular-Dynamics of $\mathrm{N}$-Alkanes, J. Comput. Phys., 1977, 23, 327-341.

53 M. S. Lee, M. Feig, F. R. Salsbury and C. L. Brooks, New analytic approximation to the standard molecular volume definition and its application to generalized born calculations, J. Comput. Chem., 2003, 24, 1348-1356.

54 M. S. Lee, F. R. Salsbury and C. L. Brooks, Novel generalized Born methods, J. Chem. Phys., 2002, 116, 10606-10614.

55 Y. Miller, B. Ma and R. Nussinov, Synergistic interactions between repeats in tau protein and Abeta amyloids may be responsible for accelerated aggregation via polymorphic states, Biochemistry, 2011, 50, 5172-5181.

56 Y. Raz and Y. Miller, Interactions between Abeta and mutated Tau lead to polymorphism and induce aggregation of Abeta-mutated tau oligomeric complexes, PLoS One, 2013, 8, e73303.

57 A. T. Petkova, W. M. Yau and R. Tycko, Experimental constraints on quaternary structure in Alzheimer's betaamyloid fibrils, Biochemistry, 2006, 45, 498-512.

58 Y. Xiao, B. Ma, D. McElheny, S. Parthasarathy, F. Long, M. Hoshi, R. Nussinov and Y. Ishii, Abeta(1-42) fibril structure illuminates self-recognition and replication of amyloid in Alzheimer's disease, Nat. Struct. Mol. Biol., 2015, 22, 499-505.

59 B. Ma and R. Nussinov, Selective molecular recognition in amyloid growth and transmission and cross-species barriers, J. Mol. Biol., 2012, 421, 172-184.

60 J. X. Lu, W. Qiang, W. M. Yau, C. D. Schwieters, S. C. Meredith and R. Tycko, Molecular structure of betaamyloid fibrils in Alzheimer's disease brain tissue, Cell, 2013, 154, 1257-1268.
61 R. P. Nanga, J. R. Brender, S. Vivekanandan and A. Ramamoorthy, Structure and membrane orientation of IAPP in its natively amidated form at physiological $\mathrm{pH}$ in a membrane environment, Biochim. Biophys. Acta, 2011, 1808, 2337-2342.

62 J. R. Brender, K. Hartman, R. P. Nanga, N. Popovych, R. de la Salud Bea, S. Vivekanandan, E. N. Marsh and A. Ramamoorthy, Role of zinc in human islet amyloid polypeptide aggregation, J. Am. Chem. Soc., 2010, 132, 8973-8983.

63 S. A. Kotler, J. R. Brender, S. Vivekanandan, Y. Suzuki, K. Yamamoto, M. Monette, J. Krishnamoorthy, P. Walsh, M. Cauble, M. M. Holl, E. N. Marsh and A. Ramamoorthy, High-resolution NMR characterization of low abundance oligomers of amyloid-beta without purification, Sci. Rep., 2015, 5, 11811.

64 J. R. Brender, J. Krishnamoorthy, M. F. Sciacca, S. Vivekanandan, L. D’Urso, J. Chen, C. La Rosa and A. Ramamoorthy, Probing the sources of the apparent irreproducibility of amyloid formation: drastic changes in kinetics and a switch in mechanism due to micellelike oligomer formation at critical concentrations of IAPP, J. Phys. Chem. B, 2015, 119, 2886-2896.

65 X. Yu, Y. Luo, P. Dinkel, J. Zheng, G. Wei, M. Margittai, R. Nussinov and B. Ma, Cross-seeding and conformational selection between three- and four-repeat human Tau proteins, J. Biol. Chem., 2012, 287, 14950-14959.

66 S. H. Shim, R. Gupta, Y. L. Ling, D. B. Strasfeld, D. P. Raleigh and M. T. Zanni, Two-dimensional IR spectroscopy and isotope labeling defines the pathway of amyloid formation with residue-specific resolution, Proc. Natl. Acad. Sci. U. S. A., 2009, 106, 6614-6619.

67 J. Nasica-Labouze, P. H. Nguyen, F. Sterpone, O. Berthoumieu, N. V. Buchete, S. Cote, A. De Simone, A. J. Doig, P. Faller, A. Garcia, A. Laio, M. S. Li, S. Melchionna, N. Mousseau, Y. Mu, A. Paravastu, S. Pasquali, D. J. Rosenman, B. Strodel, B. Tarus, J. H. Viles, T. Zhang, C. Wang and P. Derreumaux, Amyloid beta Protein and Alzheimer's Disease: When Computer Simulations Complement Experimental Studies, Chem. Rev., 2015, 115, 3518-3563.

68 M. F. Sciacca, D. Milardi, G. M. Messina, G. Marletta, J. R. Brender, A. Ramamoorthy and C. La Rosa, Cations as switches of amyloid-mediated membrane disruption mechanisms: calcium and IAPP, Biophys. J., 2013, 104, 173-184.

69 K. Hartman, J. R. Brender, K. Monde, A. Ono, M. L. Evans, N. Popovych, M. R. Chapman and A. Ramamoorthy, Bacterial curli protein promotes the conversion of PAP248-286 into the amyloid SEVI: cross-seeding of dissimilar amyloid sequences, PeerJ, 2013, 1, e5. 